\title{
Nuclear Medicine and PET/CT Technology and Techniques
}

\author{
P.E. Christian and K.M. Waterstram-Rich, eds.
}

St. Louis, MO: Mosby, 2012, 736 pages, $\$ 144$

Since its first edition in 1981, this publication has been valued as a comprehensive and up-to-date textbook on all topics within nuclear medicine. In a field that changes as rapidly as ours, it is a significant challenge to maintain that status. We are fortunate that the editors of this text have proven, time and again, to be up to that challenge.

The most significant addition to this seventh edition is chapter 12 , on the principles of MRI, coinciding with the commercial availability of PET/MRI scanners. (We can only hope that the book's title does not get lengthened yet again.) The authors of the MRI chapter are inexplicably not listed as contributors, so it is impossible to evaluate the chapter with consideration of their background. I find that they have done a pretty good job explaining the concepts of MRI at the atomic level. Pulse sequences are addressed only briefly, and the method by which the MRI signal is turned into 3dimensional image data is not discussed at all. So while the inclusion of this chapter is timely, additional material is needed for the reader to better understand this technology.

Chapter 3, on instrumentation, was completely rewritten for this edition by Mark Madsen, physicist at the University of Iowa. Both the organization and the level of detail are improved. Madsen first covers properties that can be used to describe radiation detectors, thus providing the reader with useful terminology to apply to the various instruments. He then discusses gas-filled detectors, scintillation detectors, and $\gamma$-cameras. Each section includes a description of pertinent quality control tests (the prior version of this chapter grouped these together at the end of the chapter). A final section on image quality helps the reader grasp factors that improve or degrade nuclear medicine images and includes some excellent illustrations. Single-photon emission tomography is left to chapter 9, which has not been revised (but should be, with more reference to the new version of chapter 3 ).

Many nuclear medicine technologists are just getting into PET. Chapter 10, on PET instrumentation, is quite good and is written at a level understandable to someone new to this technology. This chapter is immediately followed by a chapter on CT instrumentation, which provides excellent background for those of us who do not have direct experience with x-rays. Again, several excellent illustrations are included. And chapter 13, on clinical PET/CT, is excellent. It covers the biochemistry of ${ }^{18} \mathrm{~F}-\mathrm{FDG}$, the importance of patient preparation factors, the normal biodistribution of ${ }^{18} \mathrm{~F}-\mathrm{FDG}$, and its application to 17 different kinds of cancer. These 3 chapters can provide the learning necessary to get up to speed when one is starting to do oncology studies using PET and ${ }^{18} \mathrm{~F}-\mathrm{FDG}$.

I have always valued this book as a reference on clinical imaging, and an important and welcome addition has been made in the seventh edition. Protocols for various imaging studies are included in boxes, separate from the text. Suggestions for radiopharmaceuticals, dosage, patient preparation, instrumentation, and analysis are given for each procedure as appropriate. This material greatly enhances the value of this book as a resource.

Another significant addition is in chapter 8, on patient care and quality management, where Denise Merlino has added a section on procedure coding and billing. I have always appreciated this chapter for its wide coverage of patient care topics, and this new section provides yet another resource for my students. And I cannot forget to mention my mentor and teacher, Dr. Paul Brown, who provides an excellent review of math concepts as applied to nuclear medicine situations (chapter 1). To this day I still appreciate his straightforward explanations of complex mathematic concepts.

Nuclear Medicine and PET/CT Technology and Techniques is an excellent resource for experienced as well as student technologists. While its depth may not be satisfactory for some, its breadth in covering all aspects of nuclear medicine technology is laudable. As hard as it is to maintain the currency of a book of this style, the editors have yet again done a remarkable job.

Jennifer Prekeges, CNMT
Bellevue College
3000 Landerholm Circle SE
Bellevue, WA 98007-6484
E-mail: jennifer.prekeges@bellevuecollege.edu
Bellevue College 000 Landerholm Circle SE

COPYRIGHT @ 2012 by the Society of Nuclear Medicine, Inc.

Published online Apr. 12, 2012.

DOI: $10.2967 /$ jnmt.112.104919 